\title{
ATMOSPH (DAQ) + APP post-occupancy evaluation (POE). Energy efficiency building optimized in real time
}

\author{
Nicolás Góngora \\ Universidad Nacional del Litoral | Argentina | nicogongora@hotmail.com \\ Mauro Chiarella \\ CONICET-Universidad Nacional del Litoral | Argentina | maurochiarella00@gmail.com
}

\begin{abstract}
Greenhouse issues in existing glass-enclosed buildings can be controlled by optimizing energy efficiency and thermal comfort using low cost, customizable, customizable, open source, transferable resources. For such objectives, it is necessary to strategically link algorithmic, heuristic and manufacturing processes. For the case study, the creation of a personalized data acquisition device (DAQ) and a post-occupational evaluation APP (POE) enabled us to advance on real-time building energy efficiency operating on the need for comfort in the rooms and users.
\end{abstract}

Keywords: data acquisition; post-occupancy evaluation; domotic; arduino; architectural skin

\section{INTRODUCCIÓN}

La investigación analiza la problemática de efecto invernadero en el interior de un edificio ubicado en la ciudad de Santa Fe, Argentina. El caso de estudio Hotel UNL-ATE, es un edificio de media densidad que presenta gran porcentaje de envolventes vidriadas en la totalidad de sus fachadas y que carece, en el epicentro del problema, de ventilaciones al exterior. Investigamos con la necesidad de dar respuesta mediante una solución innovadora de bajo costo (transferible, adaptativa y de código abierto) que reconozca la necesidad de confort y eficiencia energética edilicia, en tiempo real, de los ambientes y de los usuarios.

\section{PROBLEMA}

Está comprobado en forma científica y empírica que la incidencia de la radiación solar directa sobre las envolventes acristaladas produce un notable incremento de temperatura en el interior de los ambientes afectando a confort térmico edilicio. La aplicación de fachadas muro cortina en arquitecturas construídas en clima subtropica húmedo, traen un difícil control de la radiación solar. En e mercado, si bien hay muchas soluciones pasivas aplicadas en sistemas de aberturas para materializar estas fachadas, no son lo suficientemente eficientes para nuestro contexto en particular. En estas tecnologías citamos por ej. al doble vidriado hermético y los perfiles con puentes térmicos. En este caso la ganancia de temperatura por radiación solar es tan elevada, que excede a la protección que brindan estas herramientas ya que la atenúan muy levemente. Las soluciones convencionales y generalizadas de pieles pasivas arquitectónicas, son respuestas homogéneas que no atienden a las diferentes variables y situaciones que transcurren diariamente en un edificio. La falta de adaptación contextual y personalización en tiempo real de las mismas produce pérdida de control en: el deslumbramiento, visuales interiores-exteriores, lluminación diferencial por actividades y usos, temperaturas interiores, produciendo un aumento innecesario e irracional de energía por uso de sistemas complementarios para lograr rango de confort.

\section{OBJETIVOS}

Para afrontar al problema enunciado nos propusimos desarrollar una solución innovadora como respuesta al efecto invernadero que se produce en el interior de los ambientes con envolventes vidriadas. Esta solución debía estar comunicada en tiempo real con los fenómenos físicos ambientales y con los requerimientos cualitativos de uso que necesitan los usuarios para su confort. Como objetivos particulares teníamos:

- Reconocer la problemática en un caso de estudio en la ciudad de Santa Fe, y su factor de repetición en otras obras con características similares.

- Diagnosticar cuantitativamente el problema con mediciones de adquisición de datos de manera gráfica.

- Relevar la percepción de los usuarios afectados por este problema.

- Fijar estrategias de intervención para una mejor solución del problema.

\section{METODOLOGÍA}

La metodología utilizada para el relevamiento de campo, fue la recopilación de datos cuantitativos en el edificio Hotel UNL-ATE, a través de sensores (sistemas adquisición de datos Atmosph), diseñado ad hoc. Los datos cualitativos de confort fueron relevados por encuesta a través de una rutina implementada en el mismo edificio. La misma consistía en la exposición de los encuestados a permanecer en la zona donde hemos detectado la mayor manifestación del problema, con los datos cuantitativos. Con este relevamiento de campo se logró delimitar el problema, conocer los máximos y mínimos, lo cual ha permitido establecer los márgenes de trabajo. 


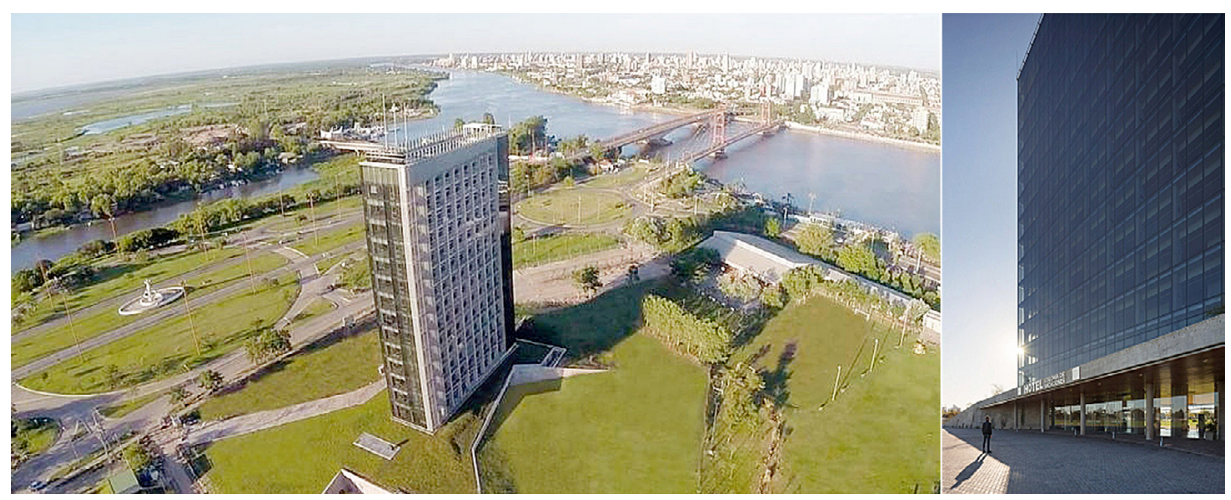

Figura 1: Caso de Estudio: Hotel UNL-ATE

Como propuesta hemos desarrollado una piel dinámica programable open source, en formato de ventana, que sirve como método de reemplazo de las existentes. Nuestro diseño de abertura incorpora sensores para tener conocimiento en tiempo real de las variables (temperatura, humedad relativa, sensación térmica, velocidad de viento). Esta información es leída a través de una placa adquisición de datos similar a arduino llamada wemos, que tiene funciones wifi. Esta placa permite alojar una web, aplicación móvil en su interior, para que los usuarios puedan conectarse con sus medios portátiles, mediante su red wifi, e ingresar datos de percepción del confort. Con los datos de sensores que lee la placa wemos y con el ingreso de votos de confort de los usuarios (App), se procesa la información y se calculan el nivel de apertura de la ventana necesarios para lograr mejorar la situación de confort presente. La programación está diseñada para trabajar en los márgenes que presenta el problema (zona amarilla en la gráfica izquierda de la Figura 2), detectado con los análisis cuantitativos, y el movimiento de salida que emite la placa wemos (output) está representada en una maqueta mediante servos y en escala real mediante pistones electromecánicos lineales.

\section{CASO DE ESTUDIO}

Para la presente investigación, por conclusiones de trabajos previos y por relevamientos de antecedentes, hemos indentificado edificios afectados por la utilización excesiva de envolventes vidriadas, para un clima subtropical húmedo. Son edificios afectados por la problemática de bajo confort térmico. Es así como elegimos el Hotel UNL-ATE como objeto de estudio en escala real de la problemática. Es un excelente referente por su gran proporción de envolvente vidriada que presenta y por su escala de media densidad. El edificio fue un proyecto que surge de una alianza estratégica entre UNL y ATE. Su construcción fue terminada en el año 2014. Entre sus características:

- Función: Esta obra contiene la función de Hotel colonia de vacaciones. Cuenta con todos los servicios pertinentes para esta función y tiene apoyatura con salas especiales para desarrollar congresos o reuniones, actividades físicas y de ocio.

- Forma: Morfológicamente es un alzado en forma de placa, prisma, con basamento en forma de rampas ajardinadas hasta el nivel de cubierta de planta baja. El prisma, se desarrolla longitudinalmente compartiendo los mismos vectores de dirección de tránsito de la ruta 168, ubicada en frente de su fachada Sur.

- Fachadas y envolventes: La parte prismática presenta envolventes vidriadas de tipología muro cortina por completo en sus fachadas Sur, este y Oeste, aprovechando las mejores perspectivas hacia paisaje fluvial e isleño. La fachada Norte está compuesta por un juego de balcones y barandas de vidrio, morfológicamente se parece a un sistema símil brise soleil, donde las habitaciones se retiran para protección de radiación solar directa.

- Tecnología: El sistema de envolventes está desarrollado con tecnología Aluar frente integral. Es un sistema de carpintería metálica de aluminio y posee doble vidriado hermético en el $100 \%$ de las envolventes. La estructura del edificio esta materializada con $\mathrm{H}^{\circ} \mathrm{A}^{\circ}$ en concepto de planta libre, donde las columnas están ubicadas en los bordes, solo retiradas algunos centímetros de la envolvente.

- Ventilaciones: En la planta tipo, el sector de habitaciones cuenta con 2 hojas de puertas ventanas, orientadas hacia la fachada norte. La fachada sur carece de apertura de ventanas. La fachada este posee una ventana en zona de ascensor de uso privado, la escalera de esta zona tiene una envolvente no vidriada con mallado metálico, permite intercambio aire interior-exterior. La fachada oeste cuenta solo con 2 ventanas por piso. Algo importante a destacar es que las ventanas permanecen cerradas por seguridad interna en el edificio, y por presentar sistemas de bisagras débiles respecto a las grandes presiones de viento que deben soportar en caso de estar abiertas y recibir fenómeno climáticos.

\section{ATMOSPH (DAQ)}

La primera instancia de la investigación consistió en el armado de un dispositivo de adquisición de datos portátil, con guardado de datos en memoria SD, para posterior análisis gráfico en excel. La importancia de ATMOSPH es que ha sido creado con tecnología de código abierto, fabricarlo representa una muy baja inversión: cualquier estudiante de arquitectura puede construirlo siguiendo planos y utilizando los códigos generados. La creación de herramientas económicas de medición en arquitectura significa ampliar la cantidad de profesionales investigando problemas y encontrando soluciones. Por lo cual, éste fue 

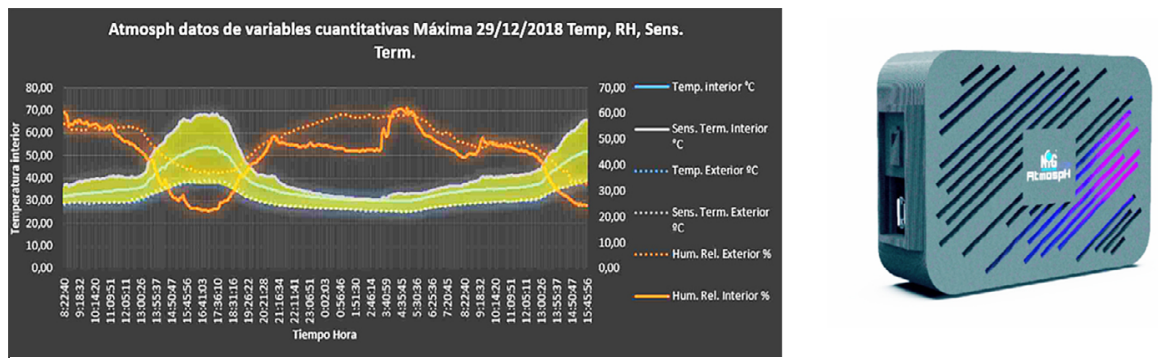

Figura 2: ATMOSPH: Dispositivo de adquisición de datos (DAQ) personalizado

uno de los objetivos de ATMOSPH: que fuera un dispositivo de bajo costo, replicable y abierto a la modificación mediante una recurrente adaptación contextual. Características:

\section{- Sistema open source}

- Microcontrolador atmega328 el mismo que arduino uno.

- Rango de medición de temperatura $-40^{\circ} \mathrm{C}$ hasta $80^{\circ} \mathrm{C}$, con sensor DHT22

- Rango de medición de Humedad relativa 0 a $100 \%$, con sensor DHT22.

- Cálculo interno por código de Sensación Térmica

- Módulo de fecha y hora

- Módulo interno para almacenar datos en tarjeta SD

- Alimentación interna con batería de celular BL-5C de $4,2 v$, recargable con cargador externo micro usb compatible celular.

- Frecuencia estándar de lectura de datos y guardado en memoria SD de 10minutos, pudiendo ampliar esta variable o disminuirla hasta $1 \mathrm{~s}$.

- Desarrollado con sistema Sleep para ahorro de energía.

- Dimensiones $92 \mathrm{~mm} \times 63 \mathrm{~mm} \times 27 \mathrm{~mm}$

- Caja plásticas imprimibles con tecnología FDM, Impresora 3d.

- Capacidad de reprogramación de software interno mediante IDE de arduino.

\section{ADQUISICIÓN DE DATOS}

Durante el tiempo que duro esta investigación, instalamos cajas recopiladoras de datos cuantitativos en el exterior $e$ interior del Hotel UNL-ATE. Esta información está compuesta por mediciones de sensores de temperatura, humedad relativa, sensación térmica, con fecha y hora de cada momento de censado. De toda la información recopilada, rescatamos a modo de síntesis para el relevamiento cuantitativo, el momento de pico máximo de temperatura interior y el momento de pico mínimo de temperatura interior. Los datos ubicados entre estos dos extremos, son valores que se repiten con mayor frecuencia entre estaciones más neutrales, otoño y primavera. El pico máximo de temperatura lo medimos el día 29 de diciembre de 2018 en donde coincidió con uno de los días más calurosos del año.

Teniendo en cuenta que la zona de análisis es la denominada "zona de estudio" (destacada en color rojo en la planta derecha superior que integra la Figura 4), el ATMOSPH que se instaló en ese sector registró una temperatura máxima de $53,60^{\circ} \mathrm{C}$. En ese mismo instante la humedad relativa fue de $22,30 \%$ y la sensación térmica de unos $67,72^{\circ} \mathrm{C}$. Este pico de temperatura se dio aproximadamente a las $17 \mathrm{hs}$ punto donde la radiación solar y el ángulo de inclinación del sol emplean la mayor ganancia térmica en la zona roja de estudio. La diferencia entre la temperatura máxima interior de $53,60^{\circ} \mathrm{C}$ y la temperatura en ese momento exterior de $34,03^{\circ} \mathrm{C}$, es de $19,57^{\circ} \mathrm{C}$. La sensación térmica máxima interior como dijimos de $67,72^{\circ} \mathrm{C}$ mantiene una diferencia de $33,06^{\circ} \mathrm{C}$, con la sensación térmica exterior presente en ese momento que fue de $34,12^{\circ} \mathrm{C}$. Estos valores muestran e problema de ganancias térmicas por radiación solar que tenemos en edificios de envolventes vidriadas. Un claro ejemplo lo tenemos también en los vehículos cuando los dejamos en el sol con los vidrios cerrados. Las temperaturas máximas a las que se llega son equivalentes a las mediciones de este edificio.
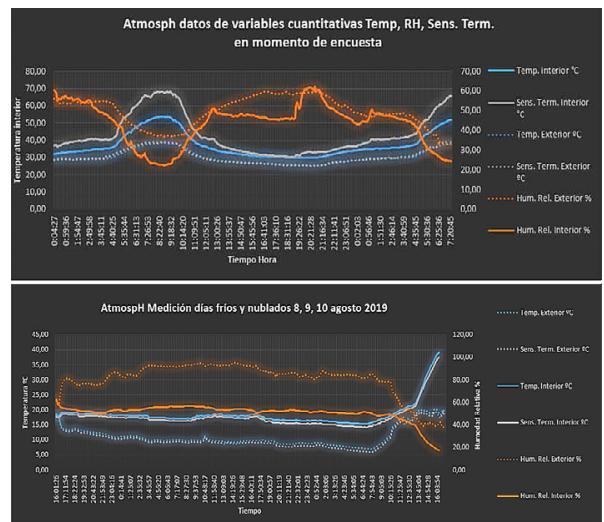

Figura 3: Mediciones temperaturas (Verano-Invierno)

Nos queda por analizar lo que acontece con las temperaturas internas en el edificio en el día más frío del año en promedio diario. El método de medición fue similar al anterior ubicando una caja en la "zona de estudio" y otra caja exterior en la terraza del Hotel. De todas las mediciones en invierno hubo días de picos más frío en el exterior, pero con alto índice de radiación solar, lo que provoca aumento espontaneo de temperatura interior con promedio diario de temperatura no tan bajo. Los días de temperatura más baja dentro del edificio, son las jornadas frías y nubladas. Los días fríos de invierno donde el sol se ve resplandeciente, provocan ganancias térmicas internas por encima de la temperatura necesaria para calefacción, y con ellas se puede almacenar calor en el interior para soportar la baja de temperaturas en primeras horas nocturnas. En los datos recopilados y graficados ver el comportamiento de la temperatura interna y externa en día nublado y frío como fue el 9 de agosto de 2019. La temperatura máxima interna registrada fue de $18,40^{\circ} \mathrm{C}$ a 
las 16:45 hs del día 9 de agosto de 2019. En ese mismo momento la temperatura exterior fue de $9,80^{\circ} \mathrm{C}$, una diferencia de temperatura de 8,6 grados entre interior y exterior. La sensación térmica, a diferencia del momento de máxima temperatura interna se encuentra $0,72^{\circ} \mathrm{C}$ por debajo de la temperatura interior.

\section{ENCUESTA PERCEPCIÓN DE USUARIO (POE)}

Para el relevamiento de datos cualitativos sobre el problema, hemos realizado encuestas ocupacionales a personal que desarrollan a diario tareas en el edificio. Como estrategia para esta encuesta sobre un problema técnico hemos creado una rutina a la cual los encuestados debían someterse antes de contestar las preguntas. La rutina consistía en que los encuestados se dirijan a la zona de estudio del edificio. Esta zona de estudio está comprendida en las proximidades de la fachada oeste y se extiende desde los pisos 2 y 13 de la placa prismática del Hotel. Este sector por sugerencia de personal de mantenimiento, fue el indicado como el más caluroso de la placa y pudimos comprobarlo con las mediciones cuantitativas. Los encuestados debían moverse caminando (Walking about Met 1,7 según TABLE A1 Metabolic Rates for Typical Tasks en ANSIASHRAE 552010), subir y bajar por ascensor a cualquiera de los pisos de estudio y permanecer 10 minutos en el área próximo a la fachada oeste. Una vez completada la rutina, descenderían a planta baja a completar el formulario encuesta web creado en google drive.

Previo a las encuestas instalamos una adquisición de datos Atmosph en el área de estudio y otra en la terraza (cielo abierto) para medir los datos cuantitativos en el mismo instante del recorrido. Las encuestas fueron tomadas a una temperatura interna en promedio de $43^{\circ} \mathrm{C}$, mientras que en el exterior, terraza, la temperatura era en promedio de $31^{\circ} \mathrm{C}$. Las preguntas en las encuestas consistieron en 3 bloques. El primer bloque era valoración del nivel de ropa de los encuestados (CLO). El segundo bloque eran preguntas relacionadas al confort en el momento. Por último el 3er bloque es en relación a las envolventes existentes.

En los resultados de las encuestas podemos observar que en el primer bloque las personas encuestadas presentaban en un $14,3 \%$ remeras mangas largas y pantalón corto un valor CLO aproximado de 0,5 . Un $42,9 \%$ de los encuestados presentaban remera mangas largas y pantalón largo, representando un CLO de 0.8. Y por último el restante $42,9 \%$ encuestado presentaban remera mangas cortas y pantalón largo representando un CLO de 0,4 . En el 2 do bloque de preguntas en relación a confort podemos destacar un $71,4 \%$ de percepción de sensación térmica en la zona de estudio como calurosa. Un $71,4 \%$ de los encuestados desean que la temperatura sea más baja. La misma proporción percibe como inaceptable la temperatura de la zona de estudio. Un $71,4 \%$ de los encuestados además ha remarcado que la sensación térmica que le produce la zona de estudio es sofocante y un $14,3 \%$ voto por altamente sofocante. También en este bloque, se ha votado sobre el nivel de brisa existente en la zona de estudio arrojando como resultado en un $57,1 \%$ que la brisa es inexistente y en el resto de las personas la brisa la percibían leve. Por último, el bloque restante de pregunta es en relación a las envolventes vidriadas del edificio ya que son el principal problema de la generación de ganancias térmicas en el interior de los edificios. Este tipo de envolventes tienen un desempeño visual desde el interior del edificio hacia el exterior que se muestra en esta pregunta de encuesta. En la misma los usuarios votaron entre 8 y 10 puntos ( 0 puntos: visuales poco atractivas, 10 puntos: visuales alucinantes)

\section{DIAGNÓSTICO Y PAUTAS DE DISEÑO}

Los relevamientos cuantitativos y cualitativos permiten confirmar la existencia del problema de radiación solar interna en el edificio Hotel UNL-ATE. En mayor proporción este problema se da en el núcleo de circulación vertical de la fachada oeste, (zona roja planta de Figura 4). Este lugar es el más estanco del edificio, con casi nula circulación de aire, y el que recibe mayor proporción de iluminación solar directa. Es necesario que este problema se transforme en una ventaja en el Hotel. Es muy fácil generar calor en días fríos con la presencia de rayos solares y por supuesto en los calurosos, gracias al efecto invernadero. El edificio no requiere en gran medida de horas de calefacción. Por otra parte, al Hotel le es muy difícil y costoso bajar las temperaturas internas que aumentan por radiación solar. Aquí es el punto más importante a intervenir. Dado que en los momentos de mayor temperatura interna en el edificio $\left(53,60^{\circ} \mathrm{C}\right.$, Figura 3$)$, en el exterior se registran temperaturas más bajas $\left(34,03^{\circ} \mathrm{C}\right.$, Figura 3$)$, es apropiado lograr intercambio de aire interior exterior para nivelar las temperaturas internas con las externas. El área sombreada amarilla (gráfico izquierdo de Figura 2), representa con precisión el margen de trabajo para solucionar el problema.

Con las tecnologías de la arquitectura bioclimática este margen de trabajo es imposible atacarlo con la eficiencia que permite la electrónica con actuación en tiempo real. Aquellas viejas soluciones sirven como una aproximación para solucionar el problema de radiación solar directa. En esos momentos, de intercambio de aire interior-exterior, deben desactivarse los sistemas de Aire acondicionado para que no trabajen sin sentido, enviando aire frío al exterior. Para lograr el intercambio de aire, es necesario cambiar la situación actual de las ventanas en el Hotel que permanecen cerradas, debido a su falta de resistencia mecánica para resistir fenómeno de viento y también por cuestiones de seguridad para los huéspedes. En el peor de los casos que se tenga una temperatura exterior y interior de $34^{\circ} \mathrm{C}$, en la zona roja de estudio, se pueden generar corrientes de aire que permitan llevar esa situación a confort. Esto lo podemos hacer diseñando unas ventanas especiales, que se comporten como pieles responsivas en tiempo real, con tecnología de código abierto y que respondan a necesidades funcionales de los usuarios. Sabiendo que la temperatura interna de aire va a ser igual a la temperatura de aire exterior, si se necesita más frío para lograr confort, la estrategia consistirá en controlar el flujo de aire que entre por las ventanas para ajustar en distintos rangos la zona de confort. Cuando se desee generar más calor se procederán a cerrar las ventanas para que la temperatura del aire empiece a incrementar por efecto invernadero. De la misma manera para protegerse de noches muy frías, se pueden cerrar las ventanas antes de que el sol caiga, para aumentar la temperatura de aire interior durante las primeras horas de la noche. Esta última estrategia disminuye considerablemente el uso de calefacción. El sistema de ventanas se puede desarrollar con tecnología arduino, y materiales que encontramos en el mercado local. 

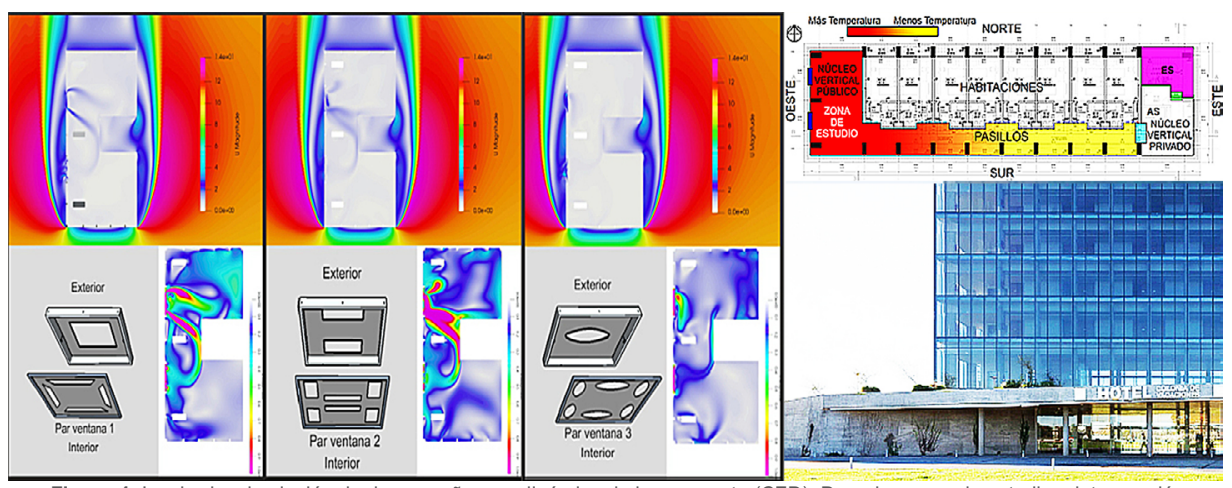

Figura 4: Izquierda: simulación de desempeño aerodinámico de la propuesta (CFD), Derecha: zona de estudio e intervención

\section{SIMULACIONES CFD Y PROPUESTA}

Mediante la adquisición de datos y encuestas de percepción de usuario hemos dimensionado la problemática estableciendo los márgenes de trabajo para solucionar el problema de ganancias térmicas internas por radiación solar. La propuesta diseñada se posicionó en los vanos de las pocas ventanas existentes, permitiendo ingresos de aire de forma controlada en tiempo real e impidiendo que esto presente riesgos de seguridad ante huéspedes. La estrategia consiste en ventilar lo máximo posible la zona de estudio (marcada en rojo, planta en Figura 4), estudiando la mejor combinación posible entre porcentajes de aperturas de ventanas y velocidad/dirección de los flujos de aire. Es decir, se desarrolla un sistema de ventanas responsivas que se accionan con un algoritmo que surge de la adquisición permantente de datos en tiempo real (DAQ) y una app específica diseñada para medir el confort térmico de los usuarios (POE), también en tiempo real. Es decir, la apertura del actuador se realiza con las variables: temperatura, votos de usuarios y velocidad de flujo de aire que pasa por la ventana. La lectura de sensores y movimiento de actuadores de las ventanas responsivas, se hacen de manera automática, gracias al hardware de soporte. Por otra parte, el ingreso de datos por votos se hace mediante una aplicación móvil de código html que se almacena dentro de una controladora wemos (la misma que se utiliza para programar las ventanas). Esta app también está programada dentro de la unidad controladora, para que sea enviada a cada usuario que se conecte a la red wifi para realizar votaciones. La placa wemos se programa a través del software de arduino IDE.

Se prueban varios diseños de apertura de ventanas responsivas y para la verificación de la eficiencia de diseño de las mismas, se realizó una simulación de desempeño aerodinámico (software OpenFoam CFDComputational Fluid Dynamics). Esta simulación nos permitió comprobar la notable mejora en el control térmico edilicio por piso, optimizando la eficiencia energética y por ende el confort de usuario (imágenes a la izquierda de la Figura 4). Para aumentar el nivel de eficiencia de manera integral y solidaria entre cada piso será necesario aumentar el nivel complejidad de la programación incorporando nuevos sensores a futuro para poder atacar otros problemas con el mismo dispositivo desarrollado.

\section{DISCUSIÓN}

La problemática de efecto invernadero en edificios existentes de envolventes vidriadas puede controlarse optimizando la eficiencia energética y el confort térmico mediante recursos transferibles, personalizables, de bajo costo, código abierto y optimizada en tiempo real. Para tales objetivos es necesario vincular estratégicamente procesos algorítmicos, heurísticos y de manufactura.

Los sistemas encargados de regular condiciones de confort en arquitectura (iluminación, ventiladores, deshumidificadores, aires acondicionados) la mayoría de las veces responden a interfaces mecánicas, teclas encendido (on) y apagado (off). Los más avanzados agregan también sensores en tiempo real para el censado de variables físicas ambientales y establecen métodos de funcionamiento a través de las necesidades atmosféricas. Pero lo que no hacen, es establecer lecturas de los requerimientos de confort que necesiten los usuarios en ese momento, son incapaces de realizar mediciones a grupos de personas para conocer su sensación de confort actual y elaborar ajustes necesarios en los individuos. Por lo tanto, este método que presentamos puede ser aplicado, no sólo a las pieles dinámicas, sino también a toda la gama de equipos electrónicos relacionados al confort de usuario.

El sistema de votos y red wifi de nuestra propuesta, debe entenderse como un método que puede aplicarse en todos los aparatos del mercado, relacionados al confort, como una función más destinada para lograr mayor eficiencia energética. Esta propuesta de ventanas puede extenderse en un futuro agregando más sensores para poder operar ante otros problemas, como pueden ser lograr aperturas mediante el control en tiempo real de índice de Co2, o lograr cierres de ventana ante principios de encendio. De esta manera se estarían agregando más funciones y más valor agregado en un mismo producto.

Si bien ha sido clave una primera simulación de desempeño aerodinámico para comprobar la notable mejora en el control térmico edilicio por piso, creemos que los ensayos aerodinamicos hay que plantearlos necesariamente de manera integral, comprobando todos los pisos del edificio, ya que la velocidad del aire se comporta de diferente manera a medida que ascendemos en altura. Sumar más información permitirá encontrar nuevas lógicas de soluciones al entender la complejidad del problema de manera global. En un futuro el sistema 

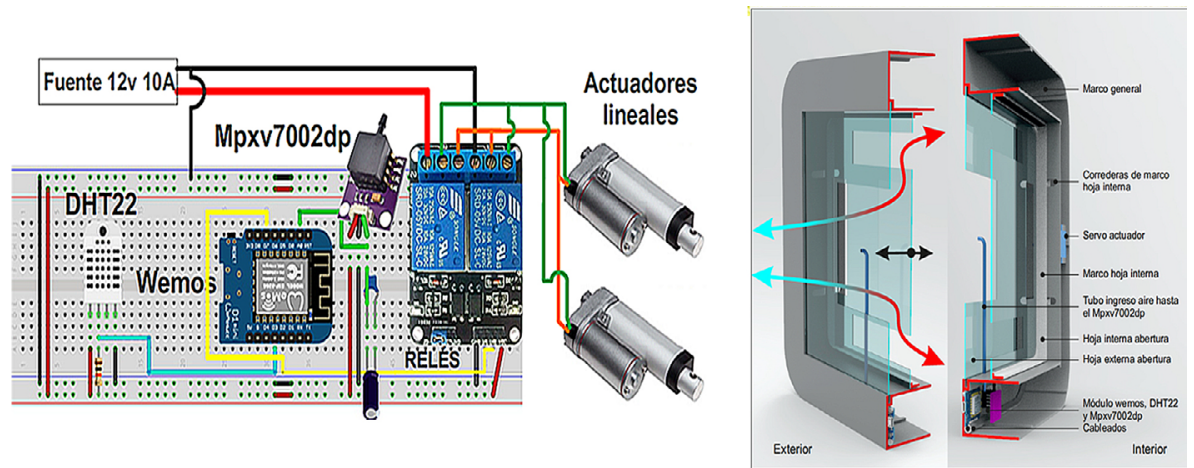

Figura 5: Izquierda, conexión del hardware usado en el prototipo (sensores, actuadores y placa wemos). Derecha, detalle construcción prototipo ventana responsiva

debería posibilitar crear redes de telemetría la cual recopile y vincule, todos los datos de las ventanas de un mismo edificio. Esto permitirá desarrollar una red de actuación solidaria e integral, además de posibilitar el envío de datos a la nube para control en tiempo real desde una oficina. Con estas conexiones, se puede monitorear a distancia el rendimiento en eficiencia energética de múltiples edificios al mismo tiempo, sirviendo para mejorar las fórmulas y algoritmos de los modelos matemáticos.

\section{AGRADECIMIENTOS}

Al Arq. Miguel Irigoyen (Ex-Rector UNL) por su colaboración en gestionar los vínculos con las autoridades y responsables del edificio Hotel UNL-ATE. A estos por brindarnos los permisos necesarios para desarrollar la investigación en su Hotel. Al Dr. Ing. electromecánico esp. en mecánica computacional Horacio Aguerre por la colaboración en el armado de los modelos matemáticos para las simulaciones de flujos. Al software libre porque sin él, el futuro sería igual al pasado.

\section{REFERENCIAS}

Chapman, A., (1977). Transmisión del calor. Madrid, ES: Interciencia
ASHRAE. (2010). ANSI/ASHRAE Standard 55-2010. American Society of Heating, Refrigerating and Air-Conditioning Engineers, Inc. Atlanta, US.

Chiarella, M; Martini, S; Giraldi, S; Góngora, N; Picco, C. (2016). Cultura Maker. Dispositivos, Prótesis Robóticas y Programación Visual en Arquitectura y Diseño para eficiencia energética. Proceedings of the 20th Conference of the Iberoamerican Society of Digital Graphics. Argentina. 2016. pp.961-968.

Hernandez, P. Usuarios Activos / Usuarios Pasivos. Estudio de la percepción del confort y de la acción de los usuarios en edificios de oficinas inmóticos de Argentina. Tesis Doctoral. Universidad del Bio-Bio. Concepción. Chile. (Defensa: junio 2014).

Hernandez, P., Trebilcok M. (2012). Ambiente inteligente, la acción e interacción del usuario con los sistemas de control en búsqueda del confort. En Proceedings of the 16th Iberoamerican Congress of Digital Graphics (pp. 91-95). Fortaleza, BR 